\title{
Propuesta del modelo de gestión de la RSU para la Universidad del Tolima*
}

\author{
Mario Enrique Uribe Macías** \\ Colombia
}

\section{Resumen}

El presente trabajo tiene como propósito proponer el modelo de gestión de la Responsabilidad Social Universitaria para la Universidad del Tolima, a partir del análisis de la percepción actual de sus stakeholders.

\section{Introducción}

Las universidades, como organizaciones de conocimiento, generan una serie de acciones que ocasionan impactos en cada una de sus partes interesadas y sobre las cuales se genera una responsabilidad. En este sentido, adelantan sus funciones sustantivas: docencia, investigación y proyección social; pero ¿cómo responden estas actuaciones a su

* La ponencia es producto del proyecto de investigación "Propuesta de un modelo de gestión de la Responsabilidad Social Universitaria para la Universidad del Tolima", financiado por la Universidad del Tolima y desarrollado por el grupo de investigación Gideut.

** Estudiante del Doctorado en Gerencia de Proyectos. Magíster en Administración. Profesor titular Universidad del Tolima. Contacto: meuribem@gmail.com 
compromiso con la sociedad?, ¿cómo le apuntan a satisfacer los intereses de sus stakeholders?

En la Universidad del Tolima, de Ibagué (Colombia), a la fecha no se ha incorporado la RSU como un enfoque estratégico y estructural, por lo cual cabría preguntarse: ¿cuál es el modelo de gestión de la Responsabilidad Social Universitaria que debe desarrollar la Universidad del Tolima, incorporado a sus funciones misionales, como respuesta a su compromiso con la sociedad y a la satisfacción de los intereses de sus stakeholders, teniendo presente las consecuencias e impactos de sus acciones?

A partir de esta problemática se desarrolló el proyecto de investigación referenciado a pie de página, y del cual se deriva esta ponencia, incorporando los resultados del análisis de los stakeholders internos que fueron producto de una investigación anterior. En el proyecto actual, se realizó el análisis de las comunidades externas: proveedores, graduados, y comunidad vecina, siguiendo la metodología sugerida por Vallaeys, De la Cruz y Sasia (2009).

En el documento se presenta inicialmente el marco teórico básico, que soporta la propuesta; luego se aborda la metodología descriptiva para el análisis de la información obtenida; posteriormente la percepción de los stakeholders externos, con respecto a la RSU en la Universidad del Tolima, desde el análisis descriptivo y el factorial; después la síntesis de la propuesta del modelo de gestión y, finalmente, las conclusiones pertinentes.

\section{Marco teórico}

Responsabilidad es "la capacidad existente en todo sujeto activo de derecho para reconocer y aceptar las consecuencias de un hecho realizado libremente" (Diccionario de la Real Academia Española, 2016), a partir del cual puede afirmarse que la responsabilidad social es el compromiso con el entorno social y ambiental, y el conjunto de respuestas que una persona (natural o jurídica) emprende frente al impacto que sus acciones generan a terceros, denominados comúnmente stakeholders. 
Estos se pueden definir como los individuos o grupos que pueden afectar o verse afectados por el logro de los objetivos de la firma (Freeman, 1984) y que, además, son vitales para la supervivencia y el éxito de la empresa (Freeman, Wicks \& Parmar, 2004). Son, en concepto de Friedman y Miles (2006, p. 4), "esos grupos sin cuyo apoyo una organización dejaría de existir".

En la misma línea, la RSU puede "entenderse como una mística que trasciende la esfera del conocimiento y la investigación para concretarse en la utilidad de la sociedad" (Rodríguez, 2012, p. 13). Con el fin de gestionarla adecuadamente, Vallaeys et al. (2009) definen cuatro tipos de impactos que genera la Universidad: organizacional, educativo, cognitivo y social; con base en ellos, surgen los ejes de gestión de la RSU: desde la organización, el campus responsable; a partir de la cognición, la gestión social del conocimiento; con base en lo social, la participación social; y desde la educación, la formación profesional y ciudadana responsable.

El mismo Vallaeys (2008, p. 209) define la RSU como

una política de mejora continua de la Universidad hacia el cumplimiento efectivo de su misión social mediante 4 procesos: gestión ética y ambiental de la institución; formación de ciudadanos responsables y solidarios; producción y difusión de conocimientos socialmente pertinentes; y participación social en promoción de un desarrollo más humano y sostenible.

\section{Metodología}

La investigación es descriptiva, pues "se reseñan las características o rasgos de la situación fenómeno objeto de estudio" (Salkind, citado por Bernal, 2000, p. 111), además es concluyente en la medida en que el análisis de la información obtenida genera conclusiones alrededor de las áreas de estudio, y finalmente, propositiva, debido a que su producto final es la propuesta del modelo de gestión de la RSU para la Universidad del Tolima. La información primaria fue obtenida mediante la aplicación de un cuestionario estructurado específico para cada una 
de las poblaciones en estudio: proveedores y egresados, seleccionados mediante muestreo aleatorio simple, y comunidad vecina, con base en muestreo no probabilístico.

En cuanto al proceso de desarrollo, se utilizó la metodología propuesta por Vallaeys et al. (2009), por lo cual la toma de la información se realizó alrededor de los cuatro ejes de gestión mencionados en el apartado anterior; desde allí se migró a la propuesta del modelo de gestión para la Universidad del Tolima, con base en el análisis estadístico realizado y a las realidades de la institución, no necesariamente reflejados expresamente en dichos ejes de gestión.

\section{Percepción de las comunidades externas de la Universidad del Tolima con respecto a las acciones y prácticas que esta desarrolla en el campo de la RSU}

A todos los stakeholders consultados se les propuso un conjunto de afirmaciones (cuadros 1, 2 y 3), sobre las cuales se les pidió que eligieran una de seis opciones de respuesta: totalmente de acuerdo, de acuerdo, parcialmente de acuerdo, parcialmente en desacuerdo, en desacuerdo, totalmente en desacuerdo. A continuación se presentan los resultados consolidados en cada caso.

\section{Análisis descriptivo}

El análisis descriptivo es presentado de manera consolidada, por cada uno de los stakeholders externo de la universidad. Cada cuadro presenta los asuntos indagados con una letra de convención con la que, enseguida, se observa la frecuencia de respuesta en la gráfica correspondiente.

- Proveedores 
Cuadro 1. Preguntas realizadas al stakeholder proveedores

\begin{tabular}{|l|l|}
\hline A & $\begin{array}{l}\text { La Universidad del Tolima responde a sus expectativas cuando celebran al- } \\
\text { gún contrato }\end{array}$ \\
\hline B & $\begin{array}{l}\text { Estoy satisfecho con los términos del contrato por parte de la Universidad } \\
\text { del Tolima }\end{array}$ \\
\hline C & Percibo un buen clima laboral dentro de la Universidad del Tolima \\
\hline D & $\begin{array}{l}\text { No existe discriminación en el acceso a los contratos con la universidad (gé- } \\
\text { nero, religión, raza u orientación política o sexual) }\end{array}$ \\
\hline E & La universidad es socialmente responsable con sus proveedores \\
\hline F & La universidad es ambientalmente responsable \\
\hline G & La universidad ha realizado contratos de forma transparente y legal \\
\hline H & $\begin{array}{l}\text { Coinciden los principios y políticas que declara la Universidad del Tolima } \\
\text { con lo que practica a la hora de realizar la contratación }\end{array}$ \\
\hline I & Entre empresa y universidad existe un trato mutuamente respetuoso \\
\hline J & $\begin{array}{l}\text { He adquirido hábitos ecológicos desde que contrato con la Universidad del } \\
\text { Tolima }\end{array}$ \\
\hline K & $\begin{array}{l}\text { La comunicación y marketing que realiza la Universidad del Tolima son so- } \\
\text { cialmente responsables }\end{array}$ \\
\hline L & La universidad me exige que sea socialmente responsable \\
\hline M & $\begin{array}{l}\text { Mi personal recibe capacitación en términos de responsabilidad social em- } \\
\text { presarial }\end{array}$ \\
\hline $\mathrm{N}$ & Existe en mi empresa libertad sindical \\
\hline$\tilde{N}$ & La universidad me exige que sea ambientalmente responsable \\
\hline O & $\begin{array}{l}\text { La comunicación y el marketing que realizan en mi empresa son socialmente } \\
\text { responsables }\end{array}$ \\
\hline P & Mi empresa es ambientalmente responsable \\
\hline
\end{tabular}

Fuente: elaboración propia.

De acuerdo con la tabulación, la figura 1 presenta la percepción de los proveedores con respecto a la responsabilidad social de la universidad, en la que se encuentra una percepción general positiva de parte de este stakeholder, debido a que en la mayoría de preguntas, las respuestas positivas (totalmente de acuerdo, de acuerdo, y parcialmente de acuerdo) superan el $90 \%$. Caso contrario se presenta en las preguntas J, L, M, y $\tilde{N}$, en las cuales las opiniones negativas oscilan entre 40 y $52 \%$. Las variables $\mathrm{J}$ y $\mathrm{M}$ corresponden a situaciones internas del proveedor, mientras que L y $\tilde{\mathrm{N}}$ son las exigencias que la universidad le hace al proveedor para que sea social y ambientalmente responsable, 
lo cual no hace parte de los términos de referencia ni los pliegos para la contratación de productos y servicios en la Universidad del Tolima.

Figura 1. Respuestas consolidadas del stakeholder proveedores

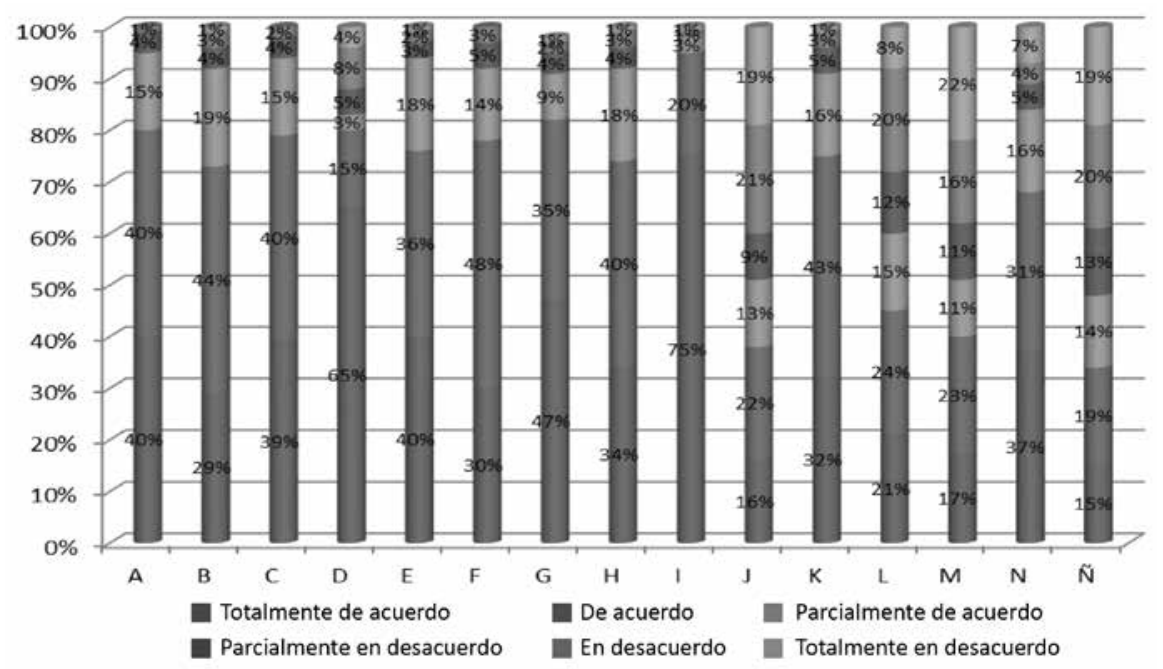

Fuente: elaboración propia.

- Comunidad vecina

De nuevo, en el cuadro 2 se presentan las preguntas realizadas con sus convenciones, en este caso a la comunidad vecina a la universidad que, como se aprecia en la figura 2, su situación es diferente. Se encuentra un gran desacuerdo con las variables propuestas mediante las preguntas aplicadas; este desacuerdo representa entre 43 y $85 \%$ del total de los vecinos, quienes no perciben que exista orientación y acciones de la Universidad del Tolima para responder con respecto a los impactos que le ha estado generando. Este es un stakeholder al cual hay que dedicarse de manera prioritaria. 
Cuadro 2. Preguntas realizadas al stakeholder comunidad vecina

\begin{tabular}{|l|l|}
\hline A & $\begin{array}{l}\text { Percibo que la universidad es socialmente responsable } \\
\text { con la comunidad vecina }\end{array}$ \\
\hline B & $\begin{array}{l}\text { Percibo que la universidad es ambientalmente responsable } \\
\text { con la comunidad vecina }\end{array}$ \\
\hline C & $\begin{array}{l}\text { Percibo coherencia entre los principios y políticas que declara la universidad } \\
\text { y lo que practica en el campus y el impacto que tiene en la comunidad vecina }\end{array}$ \\
\hline D & $\begin{array}{l}\text { La universidad realiza campañas para promover valores y temas de } \\
\text { responsabilidad social y ambiental en su comunidad }\end{array}$ \\
\hline E & $\begin{array}{l}\text { Percibo que la universidad promueve relaciones de cooperación } \\
\text { con la comunidad vecina }\end{array}$ \\
\hline F & $\begin{array}{l}\text { La universidad toma medidas para la protección del medio ambiente en el } \\
\text { campus y sus alrededores }\end{array}$ \\
\hline G & La universidad repara daños realizados a sus alrededores \\
\hline H & La universidad realiza campañas sociales en las áreas aledañas al campus \\
\hline I & La Universidad del Tolima se preocupa por los problemas sociales \\
\hline J & $\begin{array}{l}\text { He tenido reuniones con delegados de la Universidad del Tolima para tratar } \\
\text { temas de impacto social }\end{array}$ \\
\hline K & $\begin{array}{l}\text { La Universidad del Tolima interactúa con la sociedad y mide el impacto que } \\
\text { genera en ella }\end{array}$ \\
\hline L & $\begin{array}{l}\text { La Universidad del Tolima se integra con la comunidad } \\
\text { y los problemas sociales }\end{array}$ \\
\hline M & $\begin{array}{l}\text { Percibo que la universidad se interesa por los propósitos del barrio y las pro- } \\
\text { puestas sociales que se puedan generar dentro de él }\end{array}$ \\
\hline N & $\begin{array}{l}\text { La Universidad del Tolima tiene proyectos que impacten social o ambiental- } \\
\text { mente la comunidad donde resido }\end{array}$ \\
\hline
\end{tabular}

Fuente: elaboración propia. 
Figura 2. Respuestas consolidadas del stakeholder comunidad vecina

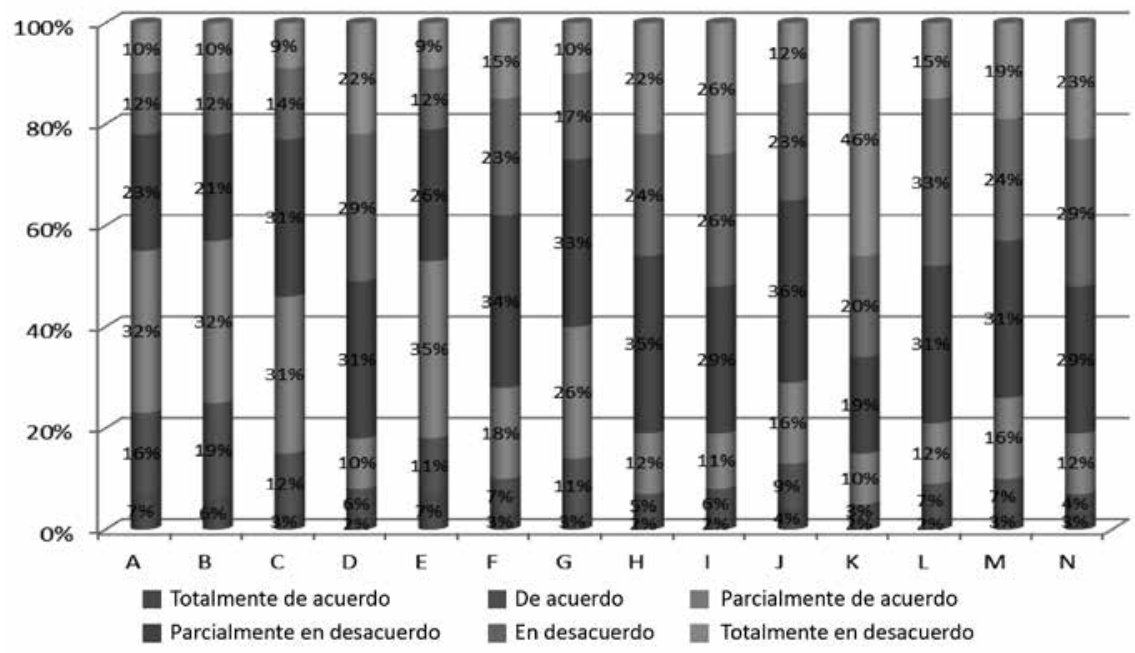

Fuente: elaboración propia.

\section{Graduados}

Ahora, en el cuadro 3 pueden apreciarse las cuestiones que respondieron los graduados, y sus convenciones; y posteriormente, los resultados de sus respuestas (figura 3), en ella se observa una opinión positiva, que fluctúa entre 94 y $99 \%$, con respecto a las preguntas A, B, C, y D, lo cual reconoce la formación integral, social y ambiental recibida de parte de la universidad. Existe otro conjunto de preguntas con apreciación positiva, constituido por los ítems E, G, H, I, y L, que arroja un rango entre 57 y $76 \%$ de las respuestas totales; por el contrario, las demás variables fueron calificadas con apreciaciones negativas, con frecuencias entre 63 y $88 \%$. Resulta evidente la necesidad de trazar una estrategia clara con respecto a los graduados, desde mucho antes que lo sean, a través de su paso como estudiantes de la universidad. 
Cuadro 3. Preguntas realizadas al stakeholder graduados

\begin{tabular}{|l|l|}
\hline A & $\begin{array}{l}\text { La Universidad del Tolima me brindó formación ética y ciudadana } \\
\text { que me ayuda a ser un profesional socialmente responsable }\end{array}$ \\
\hline B & $\begin{array}{l}\text { Mi formación fue realmente integral, humana y profesional, no solo } \\
\text { especializada }\end{array}$ \\
\hline C & $\begin{array}{l}\text { Mi formación me permite ser un ciudadano activo en defensa del me- } \\
\text { dio ambiente }\end{array}$ \\
\hline D & $\begin{array}{l}\text { La universidad me motivó para ponerme en el lugar de otros y re- } \\
\text { accionar contra las injusticias sociales y económicas presentes en mi } \\
\text { contexto social }\end{array}$ \\
\hline E & $\begin{array}{l}\text { Dentro de mi formación tuve la oportunidad de relacionarme cara a } \\
\text { cara con la pobreza }\end{array}$ \\
\hline F & $\begin{array}{l}\text { Dentro de mi paso por la universidad tuve la oportunidad de partici- } \\
\text { par en proyectos sociales fuera de la universidad }\end{array}$ \\
\hline G & $\begin{array}{l}\text { Dentro de mi vida laboral y profesional he tenido la oportunidad de } \\
\text { participar en proyectos sociales }\end{array}$ \\
\hline H & $\begin{array}{l}\text { Dentro de mi formación tuve la posibilidad de conocer a especialistas } \\
\text { en temas de desarrollo social y ambiental }\end{array}$ \\
\hline I & $\begin{array}{l}\text { Dentro de mis cursos realizados en la Universidad del Tolima tuve la } \\
\text { oportunidad de hacer investigación aplicada a la solución de proble- } \\
\text { mas sociales y/o ambientales }\end{array}$ \\
\hline J & $\begin{array}{l}\text { Percibo que los egresados están bien informados acerca de las injusti- } \\
\text { cias sociales y los riesgos ecológicos del mundo actual }\end{array}$ \\
\hline K & $\begin{array}{l}\text { Se han realizado reuniones con egresados por parte de la Universidad } \\
\text { del Tolima para discutir la adecuación del currículo a las demandas } \\
\text { sociales actuales }\end{array}$ \\
\hline L & $\begin{array}{l}\text { La universidad mantiene contacto conmigo y me ofrece algunos ser- } \\
\text { vicios desde mi graduación }\end{array}$ \\
\hline M & $\begin{array}{l}\text { La universidad me ofrece información acerca de oportunidades labo- } \\
\text { rales }\end{array}$ \\
\hline N & $\begin{array}{l}\text { La universidad me ofrece información acerca de ofertas académicas } \\
\text { que ayudan a mi crecimiento profesional }\end{array}$ \\
\hline
\end{tabular}

Fuente: elaboración propia.

\section{Análisis factorial}

Para efectos de hacer más práctico el trabajo de elaboración del modelo de Rsu para la Universidad del Tolima, se procedió a reducir el número de variables consideradas en todas las poblaciones objeto de estudio, a unas pocas dimensiones denominadas variables latentes, hipotéticas 
o constructos, con una pérdida mínima de información. En este sentido, Valderrey argumenta que:

Al observar muchas variables sobre muestras de gran tamaño es presumible que una parte de la información recogida pueda ser redundante o que sea excesiva, en cuyo caso los métodos multivariantes de reducción de la dimensión tratan de eliminarla. Estos métodos combinan muchas variables observadas para obtener pocas variables ficticias que las representen con la mínima pérdida de información. (Valderrey, 2010, p. 147)

Figura 3. Respuestas consolidadas de los stakeholders graduados

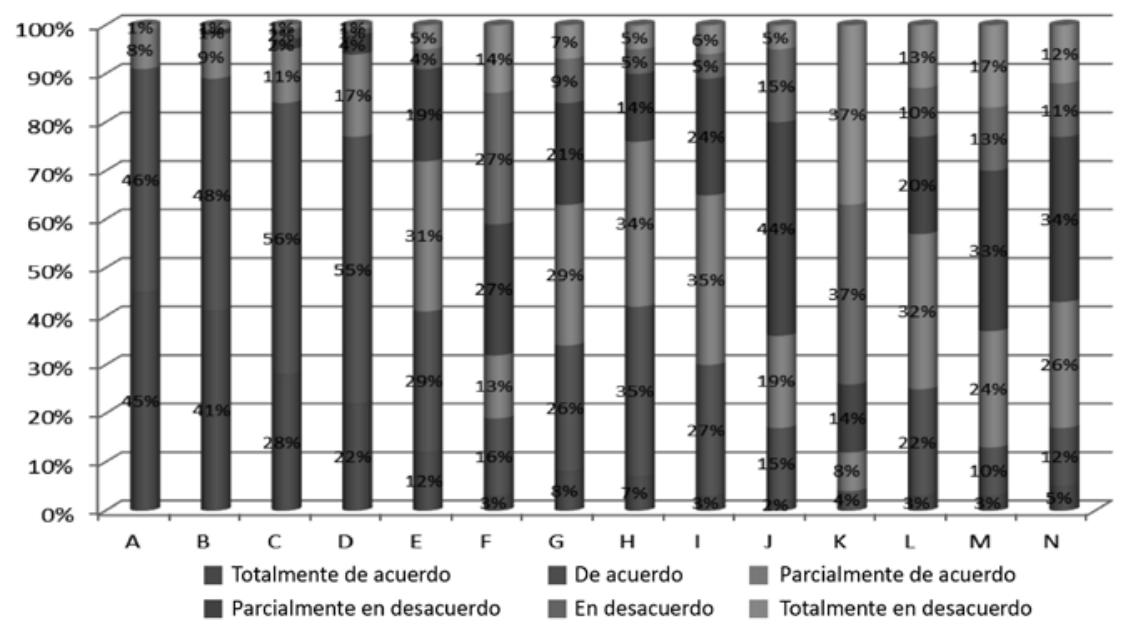

Fuente: elaboración propia.

La reducción de las dimensiones se hizo a través de las técnicas de análisis factorial por componentes principales y el escalamiento multidimensional del análisis multivariante. Kinnear y Taylor (1989) definen el análisis factorial como un procedimiento a través del cual se toma un gran número de variables, con el propósito de expresarlas en pocos factores que expliquen su intercorrelación. Para Valderrey (2010), se trata de un método de reducción de la dimensión que busca simplificar las múltiples y complejas relaciones entre un grupo de variables observadas, y de esta manera hallar constructos comunes 
denominados factores, que asocian a los atributos aparentemente incorrelados (Pérez, 2009).

Por su parte, Ferrán (2001) plantea que este análisis es una técnica que permite representar las variables en un espacio de pequeña dimensión facilitando la interpretación de las relaciones existentes entre ellas. A su vez, Quesada (2013) manifiesta que este permite la reducción de datos para encontrar grupos homogéneos y describir la asociación entre las categorías de cada variable (De la Garza, Morales y González, 2013).

Como resultado de la aplicación de la técnica, se obtuvo el conjunto de variables latentes y observables, que se pueden apreciar en el cuadro 4, y que serán determinantes para la propuesta general del modelo de gestión de la Rsu.

Cuadro 4. Variables latentes y número de variables observables

\begin{tabular}{|l|c|}
\hline \multicolumn{1}{|c|}{ Variable latente } & $\begin{array}{c}\text { No. de variables } \\
\text { observables }\end{array}$ \\
\hline Investigación, divulgación y desarrollo social & 8 \\
\hline Sensibilidad social y ambiental & 13 \\
\hline Política ambiental & 10 \\
\hline Clima laboral & 5 \\
\hline Comunicación y marketing socialmente responsables & 5 \\
\hline
\end{tabular}

Fuente: elaboración propia.

Para la definición de estas variables, se adicionaron los resultados de la consulta realizada con las comunidades internas: estudiantes, profesores, investigadores, administrativos, directivas académicas; que fueron producto del proyecto de investigación La responsabilidad social universitaria en la Universidad del Tolima: estado actual y perspectivas (Araque, Rubio y Uribe, 2015).

\section{Propuesta del modelo de gestión de la RSU para la Universidad del Tolima}

El modelo de gestión de la Rsu que se propone como resultado de la investigación desarrollada está basado en el enfoque de procesos. Un 
proceso es "un conjunto de actividades secuenciales o paralelas que ejecuta un productor, sobre un insumo, le agrega valor a éste [sic] y suministra un producto o servicio para un cliente externo o interno" (Agudelo y Bolívar, 2007), en lo cual estos autores coinciden con Camisón, Cruz y González (2007), quienes agregan que las salidas (producto o servicio) deben ser útiles para el cliente. Oakland (1989, pp. 843-844) establece que las entradas de un proceso "incluyen operaciones, métodos o acciones [...] y las salidas se dan en forma de productos, información, servicios o en general, resultados".

En consecuencia, el enfoque basado en procesos puede entenderse como "la aplicación de un sistema de procesos dentro de la organización, junto con la identificación e interacciones de estos procesos, así como su gestión para producir el resultado deseado" (Instituto Colombiano de Normas Técnicas y Certificación, Icontec, 2008, p. ii). Este enfoque tiene como ventaja principal que se puede establecer un sistema de continuo control de las relaciones y las interacciones entre los diferentes procesos de la organización.

Adicionalmente, la gestión por procesos, en palabras de Camisón et al. (2007, p. 863), "es una práctica que consiste en gestionar integralmente cada uno de los procesos que tienen lugar en la empresa, y no únicamente los procesos productivos o relativos al área de ventas, como tradicionalmente se ha venido haciendo". De acuerdo con Pérez (1993), "gestionar es hacer adecuadamente las cosas, previamente planificadas, para conseguir objetivos (comprobando posteriormente el nivel de consecución)".

En la figura 4 se presenta el esquema general del modelo que se propone, en el cual se evidencia la utilización del enfoque basado en procesos, que ha sido anteriormente definido.

Este modelo se inicia a partir de la identificación por parte de la Universidad del Tolima, de los intereses de cada uno de sus stakeholders; esta actividad exige previamente definir claramente quiénes son ellos, específicamente para la universidad. Con base en dichos intereses se genera la dinámica de los procesos relacionados con la RSU. Los procesos corresponden a las variables latentes obtenidas mediante la aplicación del análisis factorial (cuadro 4). 
Para el adecuado desarrollo de estos procesos, se requiere la definición de las políticas, que constituyen un marco de pensamiento para la acción; de las estrategias, que indican la manera como deben ser alcanzados los propósitos del modelo; de los objetivos, que son resultados a alcanzar; de los proyectos, que son la unidad autosostenida de planificación, presupuestación y ejecución de las diferentes iniciativas que se desprendan de las definiciones anteriores; de las herramientas, que constituyen los instrumentos operacionales que complementan todo el marco de actuación; de los indicadores, que son medidas gerenciales para monitorear el desempeño organizacional del modelo; y de la estructura, que corresponde al soporte institucional para la asignación de responsabilidades con respecto al modelo de gestión de la RSU.

Figura 4. Esquema general del modelo de gestión de la RSU para la Universidad del Tolima

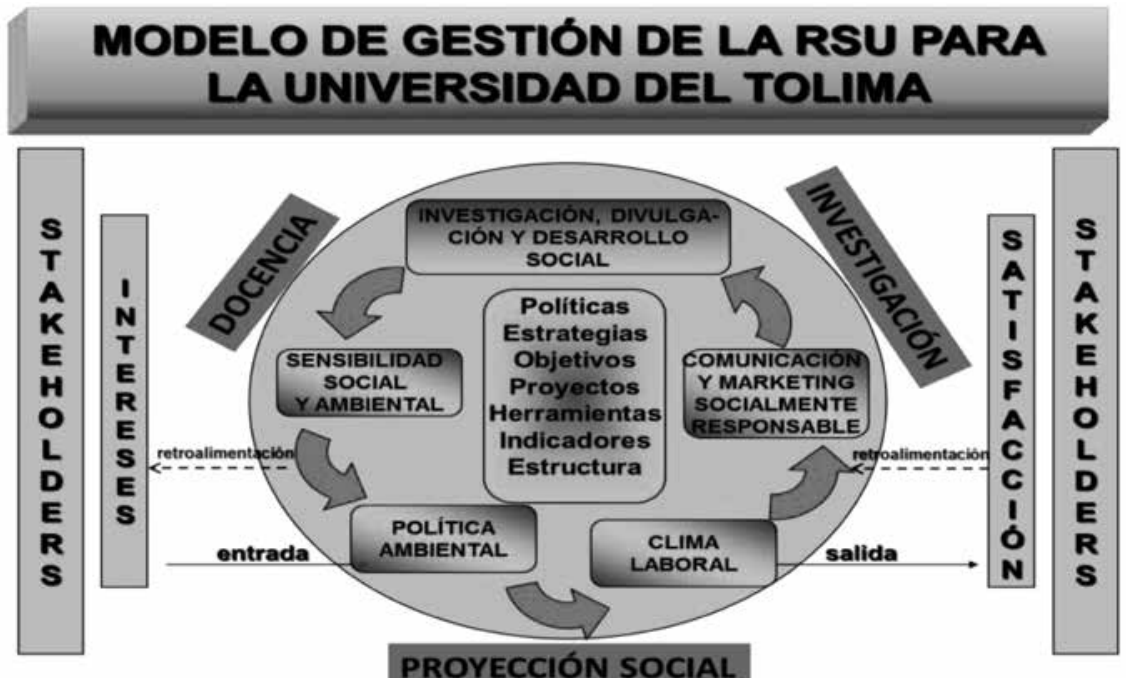

Fuente: elaboración propia.

Como se observa en la figura 4, el desarrollo de los procesos al igual que el conjunto de definiciones anteriormente expresadas, están orientados a brindar satisfacción a los intereses de los diferentes stakeholders de la universidad; es decir, el enfoque basado en procesos se origina a partir de los intereses de estos y finaliza con su satisfacción. Los 
procesos, sus interrelaciones y sus interacciones están enmarcados dentro de las tres funciones sustantivas de la universidad, que constituyen sus ejes misionales: la docencia, la investigación y la proyección social.

Es decir, el modelo de gestión que se propone no es algo diferente ni paralelo al accionar institucional; por el contrario, es una forma de gestionar la responsabilidad social que emerge, se nutre y a su vez alimenta las diferentes acciones que en el ejercicio de su objeto social desarrolla la universidad.

Es propio del enfoque basado en procesos escuchar la voz de los diferentes stakeholders, con el objeto de mejorar continuamente todo lo que se hace; por esta razón, existe un torrente de retroalimentación con base en los resultados de los procesos y a partir de la opinión expresada por todas las partes interesadas. Adicionalmente la universidad, mediante el desarrollo de sus procesos, genera la capacidad de retroalimentar a los stakeholders en cuanto a los intereses que estos tienen en la universidad, por lo cual las acciones de comunicación son muy importantes.

Lo anterior es una descripción del modelo desde la óptica estratégica. Además, se requiere generar la operación del modelo, mediante decisiones y formulaciones de los aspectos claves, como los que se presentan en la figura 5 .

En este sentido, la formulación de políticas resulta ser el primer paso. Se proponen políticas específicas para la gestión de la RSU en la Universidad del Tolima, además de las que se encuentran en su Plan de Desarrollo, en lo institucional (políticas: curricular, de educación a distancia, de educación mediada por las TIC, editorial, de internacionalización, ambiental, y de personal); en la docencia; en la investigación; y en la proyección social.

Como complemento se proponen las estrategias institucionales, de docencia, de investigación, y de proyección social. Los objetivos también se enmarcan en las mismas categorías anteriormente mencionadas. Finalmente, los proyectos serán la unidad operacional y de asignación de recursos institucionales, para evidenciar la Rsu dentro del quehacer institucional, utilizando para ello diversas herramientas administrativas que le son aplicables. 
Con el objeto de mejorar en la gestión de la RSU y en concordancia con el enfoque basado en procesos, se proponen un conjunto de indicadores que produzcan el torrente de retroalimentación para los planes de mejoramiento y de mantenimiento que se requieran.

Figura 5. Soporte de planeación y operación del modelo de gestión de la RSU

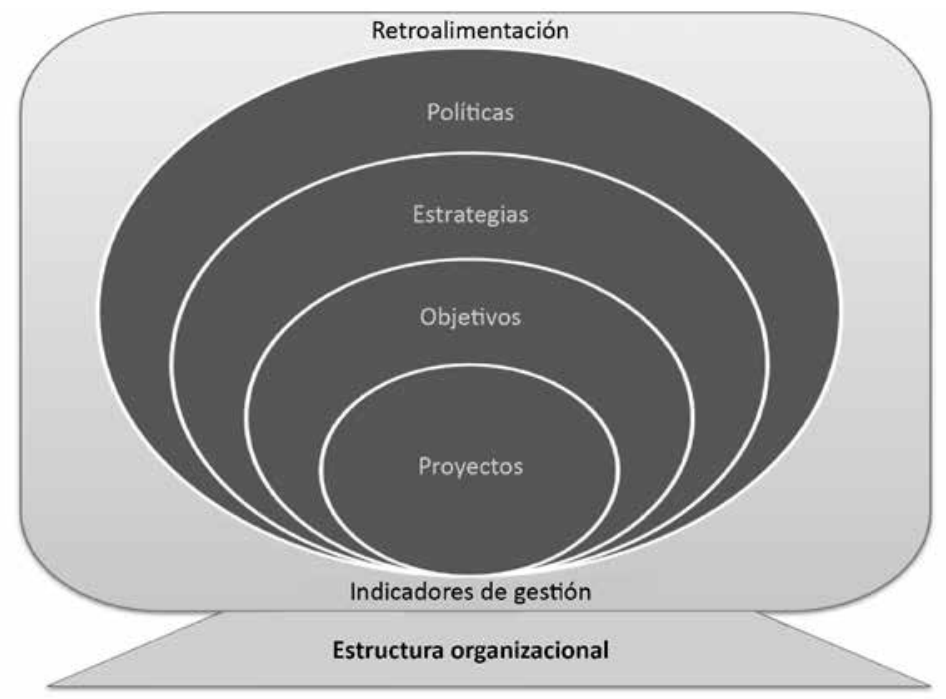

Fuente: elaboración propia.

La propuesta descansa sobre la estructura organizacional de la universidad, con una coordinación adscrita y dependiente de la Vicerrectoría Académica, en consulta con la situación actual de tipo presupuestal y financiera, que sea el motor de la gestión y el contacto con las unidades académicas (facultades, Instituto de Educación a Distancia, y programas).

\section{Conclusiones}

La RSU es una mística y una política de mejora continua, orientada a que la universidad genere impactos positivos y minimice los negativos, 
sobre sus stakeholders, externos e internos, enmarcados en los cuatro ejes de responsabilidad social que le son propios.

Con respecto a la percepción de los stakeholders externos, en cuanto a la RSU de la Universidad del Tolima, los proveedores manifiestan una opinión mayormente positiva (alrededor de $90 \%$ en la mayoría de casos), mientras que las comunidades vecinas la sienten como mayormente negativa (hasta en $85 \%$ ). Los graduados tienen, en conjunto, opiniones divididas que hacen que en algunos ítems califiquen mayormente positivo (con tendencia a $99 \%$ ) y otros mayormente negativos (cercanos a $88 \%$ ).

Estos resultados demuestran que no existe un modelo de gestión de la RSU en la Universidad del Tolima, y que esta no ha sido integrada a su diseño estratégico, como un asunto prioritario que es básico para la sostenibilidad en su escenario futuro.

Se proponen cinco variables latentes, producto del análisis factorial, que se convierten en los procesos centrales del modelo de gestión que se propone: investigación, divulgación y desarrollo social; sensibilidad social y ambiental; política ambiental; clima laboral; y, comunicación y marketing socialmente responsables.

El modelo de gestión de la RSU que se propone está basado en el enfoque de procesos, el cual incluye de manera sistémica los requerimientos previos y la posterior satisfacción de los intereses de los stakeholders, los procesos anteriormente mencionados, la formulación de las variables estratégicas y de estructura, y los procesos de retroalimentación permanente, para la mejora continua del sistema.

Esta propuesta es un esfuerzo encaminado a que la RSU sea reconocida como un asunto estratégico y de máxima importancia en la Universidad del Tolima, y de esta manera sea involucrada en la planeación y en el ejercicio del quehacer institucional, alrededor de los grandes objetivos misionales de la institución: docencia, investigación y proyección social. 


\section{Referencias}

Agudelo, L. y Bolívar, J. (2007). Gestión por procesos. Notas de clase. Medellín: los autores.

Araque, J., Rubio, G. y Uribe, M. (2015). Responsabilidad social universitaria: teoría y aplicación. Caso de análisis: Universidad del Tolima. Ibagué: Universidad del Tolima.

Bernal, C. (2000). Metodología de la investigación para administración y economía. Bogotá: Pearson Educación.

Camisón, C., Cruz, S. y González, T. (2007). Gestión de la calidad: conceptos, enfoques, modelos y sistemas. Madrid: Pearson Educación S.A.

De la Garza, J., Morales, B. y González, B. (2013). Análisis estadístico multivariante. México: McGraw Hill.

Ferrán, M. (2001). sPss para Windows. Análisis Estadístico. Madrid: McGraw Hill.

Freeman, R. (1984). Strategic management: a stakeholder approach. Boston: Pitman.

Freeman, R., Wicks, A. \& Parmar, B. (2004). Stakeholder theory and "The Corporate Objective Revisited”. Organization Science, 15(3), 364-369.

Friedman, A. \& Miles, S. (2006). Stakeholders. New York: Oxford.

Instituto Colombiano de Normas Técnicas y Certificación, Icontec (2008). NTC-ISO 9001. Sistemas de Gestión de la Calidad. Requisitos. Bogotá.

Kinnear, T. y Taylor, J. (1989). Investigación de Mercado. Un enfoque aplicado. Bogotá: McGraw Hill.

Oakland, J. (1989). Total quality management. The route to improving performance. Oxford: Butterworth-Heinemann Ltd.

Pérez, J. (1993). Fundamentos de la dirección de empresas. Madrid: Rialp.

Pérez, C. (2009). Técnicas de análisis de datos con SPSS 15. Madrid: Prentice Hall.

Quezada, N. (2013). Estadística con SPss 20. Lima: Empresa Editora Macro.

Real Academia de la Lengua Española. (2015). Diccionario de la lengua española. $23^{\mathrm{a}}$. ed. Recuperado de http://dle.rae.es/.

Rodríguez, J. (2012). La responsabilidad social es inherente a la naturaleza y misión de la Universidad. En J. Domínguez, y C. Rama (eds.), La 
Responsabilidad Social de las Organizaciones (RSO)

responsabilidad social universitaria en la educación a distancia. Chimbote: Uladech Católica.

Valderrey, P. (2010). SPSS 17: Extracción del conocimiento a partir del análisis de datos. México: Alfaomega-Ra-Ma.

Vallaeys, F. (2008). Responsabilidad social universitaria: una nueva filosofía de gestión ética y manejo inteligente para las universidades. Educación Superior y Sociedad, 13(2), 191-220.

Vallaeys, F., De la Cruz, C. y Sasia, P. (2009). Responsabilidad social universitaria. Manual de primeros pasos. México: BID y McGraw-Hill. 